\title{
Wearable Soft Artificial Skin for Hand Motion Detection with Embedded Microfluidic Strain Sensing
}

\author{
Jean-Baptiste Chossat ${ }^{\dagger}$, Yiwei Tao*, Vincent Duchaine ${ }^{\dagger}$, and Yong-Lae Park ${ }^{\ddagger}$
}

\begin{abstract}
This paper describes the design and manufacturing of soft artificial skin with an array of embedded soft strain sensors for detecting various hand gestures by measuring joint motions of five fingers. The proposed skin was made of a hyperelastic elastomer material with embedded microchannels filled with two different liquid conductors, an ionic liquid and a liquid metal. The ionic liquid microchannels were used to detect the mechanical strain changes of the sensing material, and the liquid metal microchannels were used as flexible and stretchable electrical wires for connecting the sensors to an external control circuit. The two heterogeneous liquid conductors were electrically interfaced through flexible conductive threads to prevent the two liquid from being intermixed. The skin device was connected to a computer through a microcontroller instrumentation circuit for reconstructing the 3-D hand motions graphically. The paper also presents preliminary calibration and experimental results.
\end{abstract}

\section{INTRODUCTION}

Human hands are one of the primary means of interacting with external world in our daily lives, and they allow us to manipulate and recognize various types of tools and objects with high dexterity. For this reason, tracking of hand motions has been studied not only for understanding the intention of the owner but also for actively controlling physical devices and systems as a new type of human-computer/machine interactions that could be applied to diverse fields, such as robotics, haptics, biomechanics study, rehabilitation, healthcare and entertainment. However, in spite of a variety of devices that have been developed since the 1970s both for research and for commercial applications [1], [2], it is not easy to find a simple and inexpensive solution to detect hand motions in practice due to multiple reasons, such as relatively high development cost, low accuracy, and poor user-friendliness. Therefore, development of practical hand motion detection system has a potential but is only achievable by low-cost sensing materials that could provide relatively high accuracy and reliability on capturing complex hand's motions. Furthermore, the detection system should be compact, lightweight, mechanically simple, comfortable, and convenient to use in various environments both indoor and outdoor. We decided to explore the field of wearable sensors rather than camera-based vision systems that may be considered to be more complicated and more susceptible to

The authors are with: †École de Technologie Supérieure, Montréal, Québec, Canada and *the Department of Mechanical Engineering and the Robotics Institute and the School of Computer Science, Carnegie Mellon University, Pittsburgh, PA 15213, USA. Email: jean-baptiste.chossat.1@ens.etsmtl.ca, yiweit@andrew.cmu.edu,vincent.duchaine@etsmtl.ca,ylpark@cs.cmu.edu

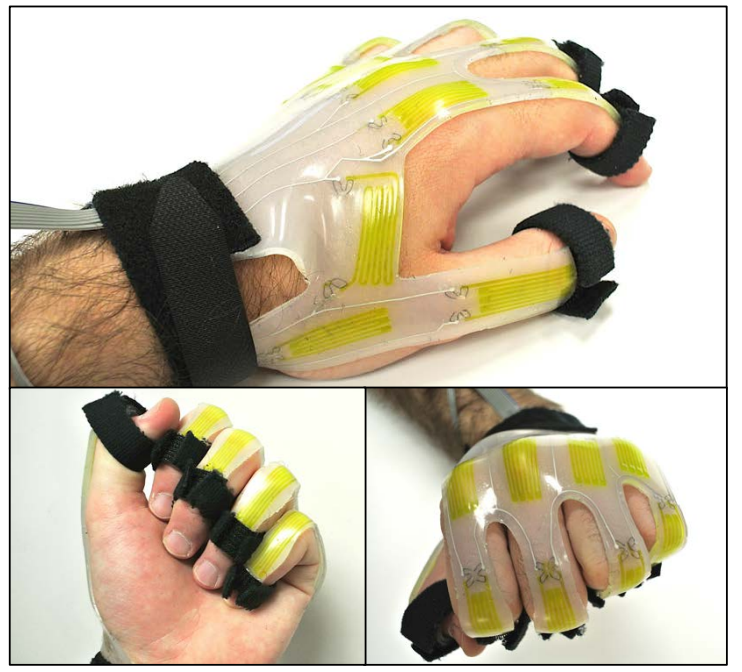

Fig. 1: Wearable soft artificial sensing skin made of a hyperelastic elastomer material for detecting multi-joint motions.

changes of sensing conditions, such as lights, users, cameras, and backgrounds [3].

Glove-based wearable sensing devices [2], [4], [5], [6] are portable systems placed on or around the hand for detecting hand gestures by measuring the finger joint motions. Various technologies have been proposed with slight functional or mechanical design differences. Although the research focus or target applications may not be always the same, many of them look like a glove that covers the entire hand.

Recent designs and commercially available devices employ different sensing mechanisms. For example, the $\mathrm{Cy}$ berGlove and the 5DT Data Glove, respectively, use optical fiber flexors and piezoresistive sensors. The key features of these technologies are easy calibration, stretchable fabrics, and a glove-like design to adapt to different hand sizes [2]. However, they are targeted at the professional market rather than the public.

However, it is well known that the palm of the hand is one the most sensitive region of our body [7], and it has also been shown that touch sensitivity and force feedback are extremely important for fine motor control and immersive virtual reality [8], [9]. Moreover, if the device is to be used for an extended period of time or to be used as an interface to execute gesture commands, it should be made as unobtrusive as possible [10]. Hence, our research is moving toward a minimalist design, covering the skin area as small as possible, as shown in Fig. 1, which will allow increased wearability and user friendliness [11]. We propose a hyper- 
elastic artificial skin with embedded conductive microfluidic channels for detecting accurate hand motions (Fig. 1).

Soft artificial skin sensors with an embedded liquid conductor have previously been proposed for multiple sensing modalities, such as strain, pressure, and shear force [12], [13], [14]. They detect the change of electrical resistance of the embedded microfluidic channels. One of the main advantages of these sensors is high stretchability and flexibility with reliable sensor responses, which make them easy to conform to a complicated 3-D shape. Also, due to the electrical and mechanical simplicity, it is easy to manufacture a device with a dense array of sensing elements in a limited area. Using this technology, soft sensing suits have been developed for measuring lower body motions [15] and wearable devices for hand motion detection [16], [17]. However, these devices employ only one liquid conductor for both sensing and wiring which makes it difficult to differentiate the resistance changes between the sensing elements and the soft wires. Also, the hand devices have a limited number of sensing areas that do not capture the entire hand motions.

In this paper, we present the design and manufacturing of soft artificial skin with an array of embedded soft strain sensors that can detect various hand motions. The 11 embedded microchannel sensors filled with an ionic liquid provides a capability of tracking one degree-of-freedom (DOF) motion on 11 finger joints. In addition to the ionic liquid, we used another type of conductive liquid (e.g. liquid metal) with much higher electrical conductivity for flexible and stretchable wiring. To the best of our knowledge, this is the first application of two different conductive liquids in a single wearable device. When an ionic liquid is used for sensing [18], [19], the large difference of nominal resistance between the ionic liquid and the liquid metal makes it easy to reject undesired signal disturbances caused by the soft wiring, as discussed in [18]. Furthermore, the simple design that covers only the back of the hand exposing the palm not only provides almost the same dexterity of a bare hand but also makes it easy to wear and take off the device.

The rest of the paper is organized as follows. Section II describes the design of the soft sensors, the wearable device, and the instrumentation circuit. Section III discusses the manufacturing processes. Section IV presents preliminary experimental results. We conclude with a discussion of future work that includes potential extensions of the prototype.

\section{DESIGN}

\section{A. Design Mission}

Motion sensing must be achieved through both precise and unobtrusive measures. Our design thus aimed for increasing the compactness of the device and also for improving its performance, which have not been easy to achieve simultaneously in the previous designs. Our skin was cast from a soft silicone rubber that can easily conform to a human hand. The skin contains embedded microchannels filled with liquid conductors for stretchable sensing and wiring. The liquids in the microchannels do neither introduce rigidity to the structure nor increase the size of the device.

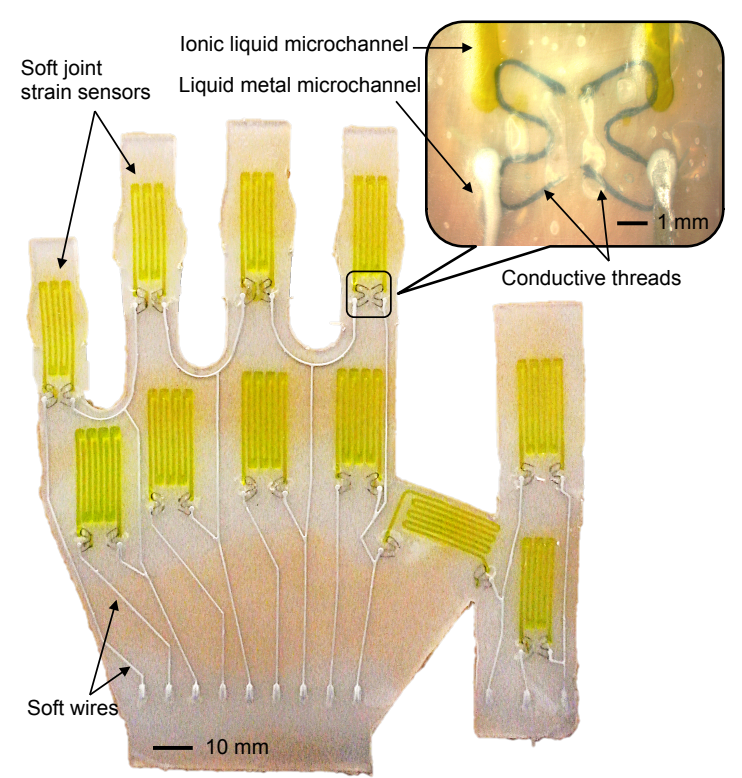

Fig. 2: Artificial skin prototype with embedded components.

\section{B. Sensor Configuration}

A human hand is highly complex and requires 24 DOFs to be fully kinematically modeled [20]. However, the concept of motor synergies [21], [22], which couple more than one DOF joint motions of one or more fingers, allows us to simplify some finger motions by defining the coupled joints. Possible reasons for the motor synergies are i) mechanically, fingers share tendons, and ii) neurophysiologically, the central nervous system simplifies the control effort for certain motions for known tasks [23]. These synergies allow us to reduce the number of necessary sensors for reconstructing general hand motions as long as the selected sensing joints are not coupled to each other [24].

We decided to place two sensors on each of four long fingers (i.e. index, middle, ring and little fingers), one on the metacarpal-phalangeal (MCP) and the other on the proximal inter-phalangeal (PIP), making total eight sensors on these four fingers. The other three sensors were placed on the thumb, the first on the inter-phalangeal (IP) joint, the second on the metacarpal-phalangeal (MCP) joint, and the third between the thumb and index finger. Fig. 2 shows the actual prototype of the skin and the sensor configuration.

With this configuration, the skin prototype can detect the flexion and extension motions of the MCP and PIP joints of the four long fingers, and also the same motions of the IP, MCP, and Carpometacarpal (CMC) joints of the thumb. In addition, the sensor between the thumb and the index finger detects abduction and adduction motions of the thumb that are important to reconstruct the hand gestures [10].

No sensors were placed on the distal inter-phalangeal (DIP) joints since the DIP joint motions can be deduced from the PIP joint motions of the same finger based on the coupling effect of the two joints [20], which simplifies our design by reducing the number of sensors and signal wires. 


\section{Detection mechanism}

1) Microfluidic sensing: Wearable devices typically require highly compliant structures to allow unconstrained body motions of the users. Although flexible or stretchable electronics technologies, such as wavy electronics and nano-particle embedded soft materials [25], are available, we decided to employ the conductive microfluidic soft sensing technique in our skin development, which allows both stretchability and flexibility of the structures. A thin elastomer layer with embedded microchannels filled with conductive liquids can be used as a highly stretchable and flexible sensor.

When placed on each joint of the hand, the microchannel of each sensor stretches with finger flexion motions. When stretched, the length of the microchannel increases and also the cross-sectional area decreases, which consequently increases the electrical resistance of the microchannel without disconnecting the circuit [12], [13].

Two different microchannels were embedded in our skin prototype with the cross-sectional areas of $700 \mu \mathrm{m}$ (width) $\times 500 \mu \mathrm{m}$ (height) for an ionic liquid and $300 \mu \mathrm{m}$ (width) $\times 500 \mu \mathrm{m}$ (height) for a liquid metal.

2) Conductive liquids: Two different conductive liquids were selected: a Room Temperature Ionic Liquid (RTIL) for strain sensing and a liquid metal for soft wiring.

RTILs, often also called as "molten salts", are liquids exclusively made of ions. These liquids are comparable to other ionic compounds such as salt. However, since the intermolecular forces binding the ions together are weak, they can maintain their liquid phases over a much lower range of temperatures. This characteristic also yields an extremely low volatility, making them suitable for being used in gas porous polymers, such as silicones. In our prototype, we filled the strain sensor microchannels with 1-ethyl-3methylimidazolium ethyl sulfate (EMISE, Sigma-Aldrich) that shows a relatively high ionic conductivity (resistivity: $\rho=25 \times 10^{-3} \Omega \cdot \mathrm{cm}$ ) and low toxicity.

Liquid metals are metal alloys that maintain their liquid phases at room temperature. In our prototype, we used eutectic gallium-indium (eGain) [26] that shows a much higher electrical conductivity (resistivity: $\rho=29.4 \times 10^{-6} \Omega \cdot \mathrm{cm}$ ) than that of the RTIL. Since typical hand motions are involved with skin stretches in multiple places on the hand, we needed to decouple finger joint motions from the skin stretches on non-joint areas for more accurate measurements. Therefore, we decided to employ a dual-liquid system in which the large resistance difference on the orders of magnitude between the two liquids rejects the signal fluctuations from the soft wiring when the RTIL is used for sensors and eGaIn for wiring.

\section{Instrumentation Circuit}

The conductivity of the RTIL is based on the movement of ions acting as charge carriers. If connected to a direct current, the ions of the RTIL will be attracted by the poles and polarize the electrodes, which prevents the sensors from generating reliable impedance values. To address this issue,

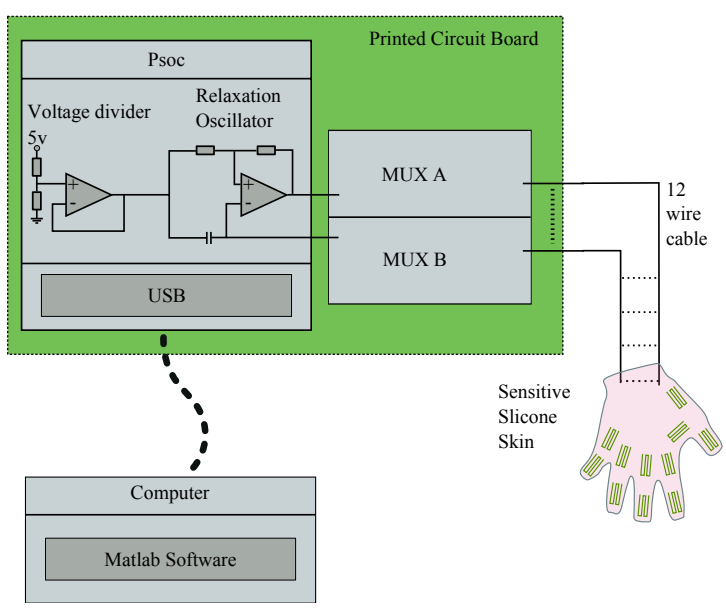

Fig. 3: System architecture of artificial skin sensing

the impedance of the RTIL was measured by applying an alternating zero-centered signal using a custom designed instrumentation circuit. The instrumentation circuit uses a Programmable system - on - Chip (PSoC - CY8C38LTI068, Cypress Semiconductor) that contains an internal micro controller and firmware programmable digital and analog components. Two operational amplifiers in this unit are used to generate a square, zero-centered, waveform whose frequency depends on the impedance of the sensor. The frequency of the waveform is then estimated within the Psoc, by a counter. Fig. 3 shows the overall architecture of our sensing and instrumentation system.

In order to switch the sensor readings between the 11 sensors, a single chip dual analog multiplexer (ADG726, Analog Devices) was used, which allowed us to selectively link the PSoC to any of the 11 skin sensors. To capture the hand motions, the multiplexer was commanded to switch the sensor readings among the 11 sensors and their impedance values were evaluated by the PSoC. Then, the sensor readings are transmitted to the computer. Since the impedance values of the sensors change the frequency of the square wave, it is not straightforward to define the sampling rate. However, using the highest possible impedance value of the sensors, we can calculate the lowest sampling rate. When switching the readings among the 11 sensors, the longest acquisition time for the entire skin would be $A=2 T \times 11$ where $\mathrm{T}$ is the maximum period of the sensor square wave, and $A$ is the acquisition time. The period is given by the equation:

$$
T=2 R_{s} C_{1} \ln \frac{1+\lambda}{1-\lambda}
$$

and

$$
\lambda=\frac{R_{1}}{R_{1}+R_{2}}
$$

where $C_{1}, R_{1}$ and $R_{2}$ are capacitance and resistances on the circuit that is used to regulate the frequency range of our square wave. In our circuit, we chose $C_{1}=1 \mu \mathrm{F}, R_{1}=22 \Omega$ and $R_{2}=100 \mathrm{~K} \Omega$, which gives $T \approx 0.44 \mathrm{~ms}$. Finally,

$$
A=11 \times T \approx 4.84 \mathrm{~ms} .
$$



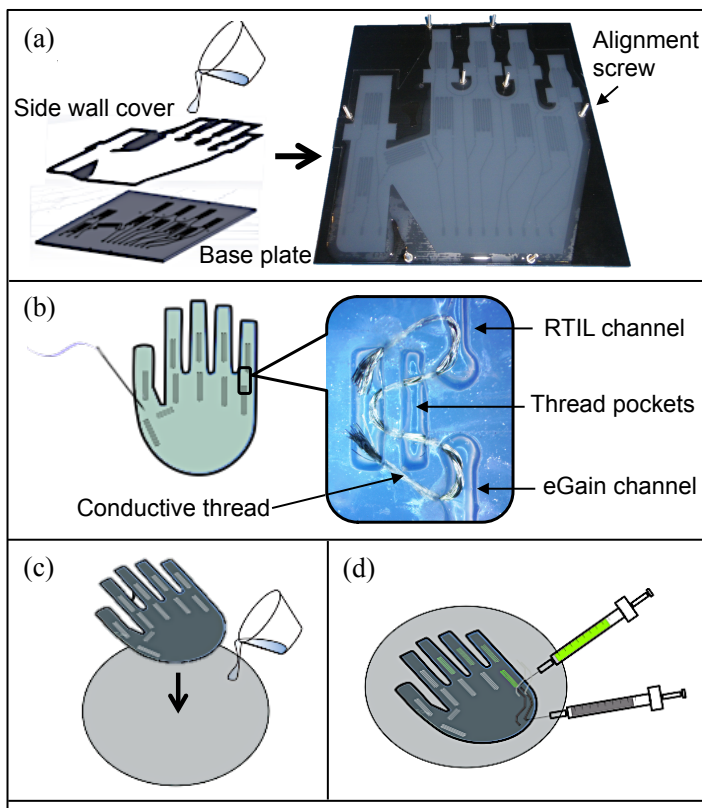

(e)

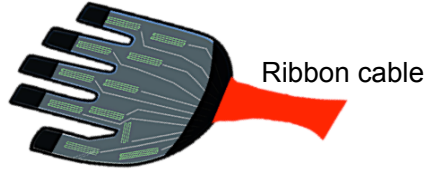

Fig. 4: Manufacturing Process for Sensing Skin: (a) Mold preparation and elastomer casting. (b) Conductive thread embedment. (c) Microchannel sealing by laminating a flat elastomer layer. (d) Liquid conductor injection. (e) Signal wire connection and attachment of finger and hand fasteners.

Therefore, the theoretical lowest sampling frequency for the entire skin is about $200 \mathrm{~Hz}$, which is much higher than the recommended frequency for hand motion detection [11].

\section{MANUFACTURING}

The manufacturing involves multiple steps of polymer casting and component embedment, as shown in Fig. 4.

\section{A. Mold Preparation}

The skin prototype is made of extremely soft elastomer (Ecoflex 00-30, Smooth-On, shore hardness 00-30, elongation at break $900 \%$ ) that is cured (at $60^{\circ} \mathrm{C}$ for approximately 25 minutes) by mixing two parts (resin and hardner, 1:1 mixed ratio in weight) in a custom-designed mold. Since the skin layer is very thin and soft, the tension of the skin does not cause any constraint (or fatigue) to the user's motion.

The mold is composed of two pieces: a 3-D printed (Objet30, Stratasys) flat base plate with protruded sensor and soft wire microchannel patterns and a laser cut (Epilog Mini, Epilog Laser) side-wall acrylic cover (1.6 mm thick) that defines the boundary of the skin to be cast. The side-wall cover is aligned and fixed to the base plate using alignment screws. The sensor channels were placed on top of the selected finger joints and the wire channels were designed to connect the sensors to the wrist.

\section{B. Skin Sensor Layer}

A liquid elastomer is poured on the assembled mold and cured in the oven at $60^{\circ} \mathrm{C}$ for approximately 25 minutes. When removed from the mold, the cured elastomer layer (1.6mm thick) contains all the microchannel patterns for strain sensing and soft wiring. Before sealing the microchannels, conductive silver threads are manually sewn into the elastomer through the pockets near the microchannels, as embedded interfaces between RTIL and eGaIn. The multiple wavy turns of the threads not only tolerate the stretch of the skin with reliable electrical connection but also prevent one liquid from invading the other. After all the thread interfaces are embedded, the sewing pockets are filled with a silicone glue (Sil-Poxy, Smoth-On, shore hardness 40A).

\section{Sealing and injection}

To seal the microchannels with the silver threads, a thin, flat base elastomer layer (approximately $800 \mu \mathrm{m}$ ) is prepared by spin-coating the same liquid elastomer at a low speed (600 rpm for $6 \mathrm{sec}$. and $350 \mathrm{rpm}$ for $25 \mathrm{sec}$.) on a flat surface. Once the flat layer is fully cured, another much thinner layer of the liquid elastomer is spin-coated at a higher speed $(1600 \mathrm{rpm}$ for $25 \mathrm{sec}$.) on top of the cured layer to be used as a bonding agent. Before curing the second layer, the original skin sensor layer is laminated on top. The two cured layers bond while the thin intermediate layer cures. A thickness of $2 \mathrm{~mm}$ was achieved over the entire area of the complete skin in this process.

When the entire skin is fully cured, conductive liquids are injected using two hypodermic syringe needles: one for injecting the liquid conductors and the other for releasing the trapped air in the microchannels.

\section{Signal Wires and Hand Fasteners}

The skin is connected to the instrumentation circuit using a 12 wire flexible ribbon cable on the wrist part of the skin. Stretchable hook and loop straps are attached to the wrist and to the ends of the fingers to fasten the skin prototype to the back of the hand reliably. The stretchable fasteners allow the user to wear and take off the skin easily without constraining the natural hand motions.

\section{Data Collection and Signal Processing}

The raw period data of 11 sensors were collected and processed using MATLAB (The MathWorks, Inc.). The mapping and 3-D graphic rendering was achieved with SynGrasp 2.0, an open source MATLAB toolbox for grasp analysis [27]. The modeling of hand adapted in SynGrasp is based on Denavitt-Hartenberg parameters. With the input joint angles, the hand gesture could be calculated from the base joint MCP joint in our model - of each finger.

For the maximal representations of hand motions that use all the 11 sensors in the skin, we construct a paradigmatic hand with 15 DOFs. The horizontal movements of the fingers were constrained in fixed angles due to the limitation of the 2-D sensing capability of our design. The two sensors at the IP and MCP joints and the one between the thumb and 


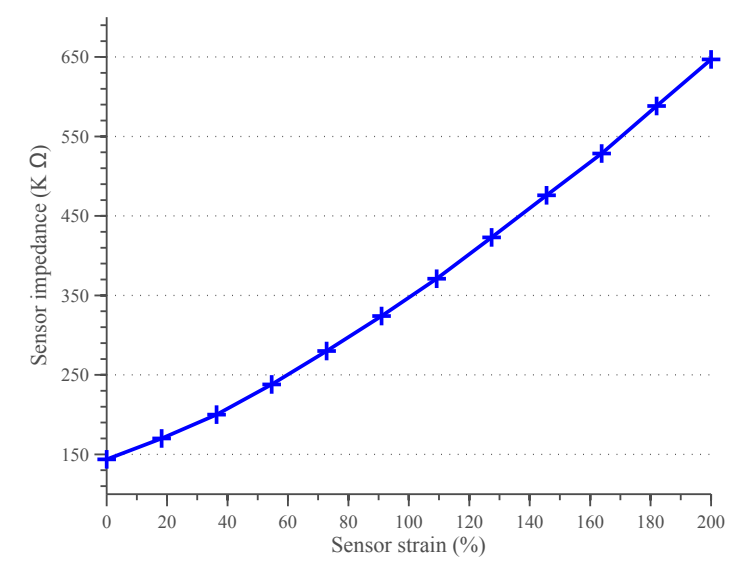

(a) Index sensor linear stretching, $4 \mathrm{~mm}$ steps up to $300 \%$ total stretch.

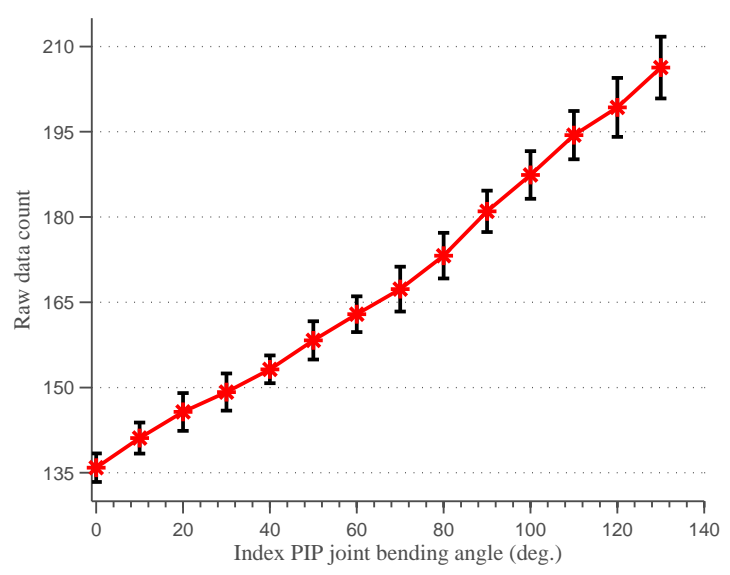

(b) Psoc raw data measure of index joint bending.

Fig. 5: Preliminary experimental results: Single axis strain test of an individual sensor (a) and finger bending test on the PIP joint of the index finger (b).

the index finger calculate the motion of the thumb in the computational model. The motions of the other four fingers are calculated by the two sensors placed on PIP and MCP joints of each finger. The IP joint motions of the four fingers are calculated synergistically from the previous PIP joints by multiplying by a factor of $2 / 3$ based on [6].

For 3-D graphical reconstruction in real-time, the skin prototype requires an initial calibration that records the sensor data of two extreme finger positions - full extension and full flexion. Then, the sensor signals are linearly interpolated to be mapped to the graphic model. The linear relationship between sensor signals and joint angles has been already verified for this type of soft strain sensors [28].

\section{Preliminary Characterization}

To characterize and evaluate the performance of our prototype, two preliminary experiments were conducted. The first experiment is calibration of an individual sensor. In this test, a single strain sensor was linearly stretched up to $200 \%$ using a commercial force/torque test stand (Mark-10 Motorized

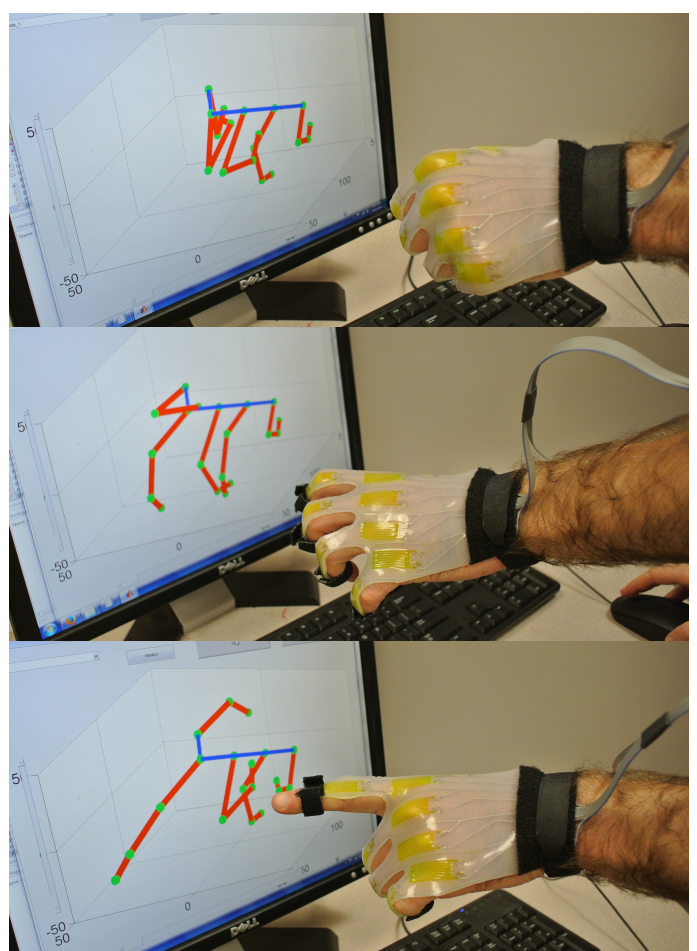

Fig. 6: Snapshots of real-time sensing system with 3-D graphical reconstruction. All five fingers (red lines) with multiple joints (green dots) rotate on the virtual anchor (blue line) based on the sensor signals from the skin.

Test Stand ESM301), and the resistance of the sensor was measured. The second experiment is calibration of the skin prototype with a hand. The skin was mounted on a human hand, and the index finger was bent from $0^{\circ}$ to $130^{\circ} 10$ times, step by step, with a $10^{\circ}$ increment. The actual angle was measured using a manual goniometer in 2-D. The results of these two experiments are shown in Fig. 5

Both strain and bending test results showed linear responses. The bending result also showed repeatability of the sensors with multiple tests. However, skin movement or slippage during the experiments was not examined in this stage. Assuming the other sensors work in the same way, these preliminary results can be extrapolated to the other joint motions except for thumb abduction-adduction. Based on the initial calibration, the skin provides joint angle information in real-time without drift, and the hand motions can be easily reconstructed without any noticeable time delays, as shown in Fig. 6.

\section{CONCLUSION}

In this paper, we presented a soft wearable sensitive skin that can detect the hand gestures and postures of the user. Our concept was directed towards a minimalist design that not only simplifies the complexity in the structure but also allows physical transparency of the user.

The artificial skin contains multiple embedded microfluidic strain sensors for detecting finger joint motions. To the best of our knowledge, this is the first attempt to use an ionic 
liquid and a liquid metal in a single wearable device, which allows rejection of signal disturbance from the soft wiring microchannels of the liquid metal. In order to interface the two different liquids, we embedded a flexible conductive silver thread between the two different microchannels of each sensor with a wavy shape during manufacturing, thus making an electrical connection of the two microchannels.

To characterize the skin sensor prototype, we conducted two experiments that calibrated a single sensor by stretching in a 2-D plane and a single finger joint by flexing and extending the joint with the skin. In the context of hand gesture and posture detection, we believe that our sensing skin showed promising preliminary results in the above two experiments in terms of linearity and repeatability. Furthermore, our proposed system overcomes the relatively high manufacturing cost and low user-friendliness shown in many of previous commercial devices.

One of the main limitations of our current prototype is the relatively complex manufacturing process. Many of the steps are involved with manual processes that require dexterity and extra care. Also, although the sealing of the microchannels was robust enough to prevent the liquids from leaking, sometimes the RTIL invaded the eGaIn microchannels following the conductive threads, consequently causing unwanted noise in the sensor reading.

In the future, we will extend our technology by improving the design that will increase the adaptability of the device to different users. Along with simplification of the manufacturing process, optimization of the fastener design and sensor placement will make the next prototype more accurate on detecting hand motions with different hand geometries. Another area will be improving the fastening mechanism, which can provide more reliable sensor signals. We will also increase the sensitivity of the sensor by further miniaturizing the size of the microchannels for increased resolution. Finally, the integration of a wireless communication unit for transmitting the sensor data to a computer or a smart device in real-time will allow a greater freedom of various applications.

\section{REFERENCES}

[1] D. Sturman and D. Zeltzer, "A survey of glove-based input," IEEE Comput. Graphics Appl., vol. 14, no. 1, pp. 30-39, 1994.

[2] L. Dipietro, A. M. Sabatini, S. Member, and P. Dario, "A survey of glove-based systems and their applications," IEEE Trans. Syst. Man Cybern. Part C Appl. Rev., vol. 38, no. 4, pp. 461-482, 2008.

[3] S. S. Rautaray and A. Agrawal, "Vision based hand gesture recognition for human computer interaction: a survey," Artif. Intll. Rev., vol. 43, no. 1, pp. 1-54, 2015.

[4] N. W. Williams, J. M. Penrose, C. M. Caddy, E. Barnes, D. R. Hose, and P. Harley, "A goniometric glove for clinical hand assessment. construction, calibration and validation," J. Hand Surg., vol. 25, no. 2, pp. 200-207, 2000.

[5] A. F. da Silva, A. F. Goncalves, P. M. Mendes, and J. H. Correia, "FBG sensing glove for monitoring hand posture," IEEE Sens J., vol. 11, no. 10, pp. 2442-2448, 2011.

[6] K. Li, I.-M. Chen, S. H. Yeo, and C. K. Lim, "Development of fingermotion capturing device based on optical linear encoder," J. Rehabil. Res. Dev., vol. 48, no. 1, pp. 69-82, 2011.

[7] P. Haggard, M. Taylor-Clark, and S. Kennett, "Tactile perception , cortical representation and the bodily self," Curr. Biol., vol. 13, no. 3, pp. 170-173, 2003.
[8] J. T. Dennerlein, D. B. Martin, and C. Hasser, "Force-feedback improves performance for steering and combined steering-targeting tasks," in Proc. SIGCHI Conf. Hum. Factors Comput. Syst. (CHI'00), (New York, NY), pp. 423-429, 2000.

[9] D. Caldwell, O. Kocak, and U. Andersen, "Multi-armed dexterous manipulator operation using glove/exoskeleton control and sensory feedback," in Proc. IEEE/RSJ Int. Conf. Intell. Rob. Syst. (IROS'95), vol. 2, (Pittsburgh, PA), pp. 567-572, Aug 1995.

[10] J. N. Ingram, K. P. Körding, I. S. Howard, and D. M. Wolpert, "The statistics of natural hand movements," Exp. Brain Res., vol. 188, no. 2, pp. 223-36, 2008.

[11] L. K. Simone, N. Sundarrajan, X. Luo, Y. Jia, and D. G. Kamper, "A low cost instrumented glove for extended monitoring and functional hand assessment," J. Neurosci. Methods, vol. 160, no. 2, pp. 335-48, 2007

[12] Y.-L. Park, C. Majidi, R. Kramer, P. Bérard, and R. J. Wood, "Hyperelastic pressure sensing with liquid embedded elastomer," $J$. Micromech. Microeng., vol. 20, no. 12, 2010.

[13] Y.-L. Park, B. Chen, and R. J. Wood, "Design and fabrication of soft aftificial skin using embedded microchannels and liquid conductors," IEEE Sens J., vol. 12, no. 8, pp. 2711-2718, 2012.

[14] D. Vogt, Y.-L. Park, and R. J. Wood, "Design and characterization of a soft multi-axis force sensor using embedded microfluidic channels," IEEE Sens J., vol. 13, no. 10, pp. 4056-4064, 2013.

[15] Y. Menguc, Y.-L. Park, H. Pei, D. Vogt, P. Aubin, L. Fluke, E. Winchell, L. Stirling, R. J. Wood, and C. J. Walsh, "Wearable soft sensing suit for human gait measurement," Int. J. Rob. Res., vol. 33, no. 14, pp. 1748-1764, 2014.

[16] J. T. Muth, D. M. Vogt, R. L. Truby, Y. Menguc, D. B. Kolesky, R. J. Wood, and J. A. Lewis, "Embedded 3D printing of strain sensors within highly stretchable elastomers," Adv. Mater, 2014.

[17] F. L. Hammond, Y. Menguc, and R. J. Wood, "Toward a modular soft sensor-embedded glove for human hand motion and tactile pressure measurement," in Proc. IEEE/RSJ Int. Conf. Intell. Rob. Syst., (Chicago, IL), September 2014.

[18] J.-B. Chossat, Y.-L. Park, R. J. Wood, and V. Duchaine, "A soft strain ensor based on ionic and metal liquids," IEEE Sens J., vol. 13, no. 9, pp. 3405-3414, 2013.

[19] J.-B. Chossat, H.-S. Shin, Y.-L. Park, and V. Duchaine, "Soft tactile skin using an embedded ionic liquid and tomographic imaging," ASME J. Mech. Rob., 2015.

[20] S. Cobos, M. Ferre, M. A. Sanch, J. Ortego, and C. Pe, "Efficient Human Hand Kinematics for Manipulation Tasks," in Proc. IEEE/RSJ Int. Conf. Intell. Rob. Syst. (IROS'08), (Nice, France), pp. 22-26, September 2008 .

[21] M. Santello, M. Flanders, and J. F. Soechting, "Postural hand synergies for tool use," J. Neurosci., vol. 18, no. 23, pp. 10105-10115, 1998.

[22] D. Prattichizzo, M. Malvezzi, and A. Bicchi, "On motion and force control of grasping hands with postural synergies," in Proc. Rob. Sci. Syst., (Philadelphia, PA), August 2006.

[23] E. Todorov and Z. Ghahramani, "Analysis of the synergies underlying complex hand manipulation," in Proc. 26th Annu. Int. Conf. IEEE Eng. Med. Biol. Soc. (EMBS'04), (San Francisco, CA), pp. 4637-4640, September 2004.

[24] M. Bianchi, P. Salaris, and A. Bicchi, "Synergy - based Optimal Design of Hand Pose Sensing," in Proc. IEEE/RSJ Int. Conf. Intell. Rob. Syst. (IROS'12), (Vilamoura, Portugal), October 2012.

[25] J.-H. Ahn and J. H. Je, "Stretchable electronics: materials, architectures and integrations," J. Phys. D: Appl. Phys., vol. 45, no. 10, p. 103001, 2012.

[26] M. D. Dickey, R. C. Chiechi, R. J. Larsen, E. A. Weiss, D. A. Weitz, and G. M. Whitesides, "Eutectic gallium-indium (EGain): A liquid metal alloy for the formation of stable structures in microchannels at room temperature," Adv. Funct. Mater, vol. 18, no. 7, pp. 1097-1104, 2008.

[27] M. Malvezzi, G. Gioioso, G. Salvietti, D. Prattichizzo, and A. Bicchi, "Syngrasp: a MATLAB toolbox for grasp analysis of human and robotic hands," in Proc. IEEE Int. Conf. Rob. Autom. (ICRA'13), (Karlsruhe, Germany), pp. 1088-1093, May 2013.

[28] Y.-L. Park, B. Chen, N. Perez-Arancibia, D. Young, L. Stirling, R. J. Wood, E. C. Goldfield, and R. Nagpal, "Design and control of a bioinspired soft wearable robotic device for ankle-foot rehabilitation,' Bioinspiration \& Biomimetics, vol. 9, no. 1, 2014. 